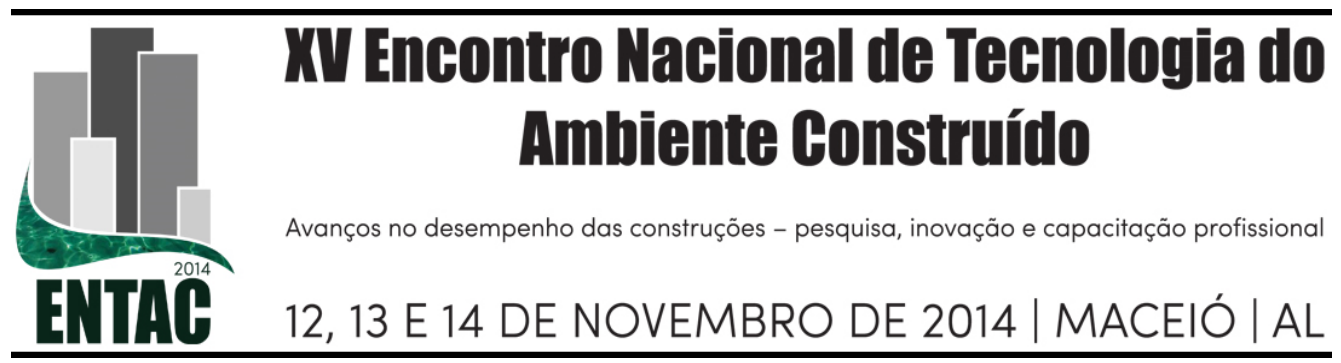

\title{
FERRAMENTA PARA PLANEJAMENTO DE DISCIPLINA BIM
}

\author{
BARISON, Maria Bernardete (1); SANTOS, Eduardo Toledo (2)
}

(1) Universidade Estadual de Londrina, tel: (43) 3371-4236, e-mail: barison@uel.br

(2) Universidade de São Paulo, tel: (11) 3091-5284, e-mail: etoledo@usp.br

\begin{abstract}
RESUMO
Devido à importância de se ensinar conceitos e ferramentas BIM (Building Information Modeling) para estudantes de Arquitetura e de Engenharia Civil, várias escolas têm procurado introduzir este assunto em seus currículos. No entanto, como o currículo já está sobrecarregado e há pouco espaço para novas disciplinas, uma estratégia útil é distribuir conteúdos BIM em diversas disciplinas do curso. O objetivo deste trabalho é propor uma ferramenta que pode ajudar professores no planejamento de disciplinas com conteúdos BIM. Este instrumento é o resultado final de uma pesquisa de doutorado em que se aplicou todo o conhecimento construído na etapa de 'Teorização'. Na pesquisa de doutorado, foi utilizada a 'Metodologia da Problematização' (MP), que possui 5 etapas: Observação da Realidade; Definição de Pontos-Chave; Teorização; Hipóteses de Solução e Aplicação à Realidade. Na etapa de Teorização, foram analisados 406 documentos com base no método 'Análise de Conteúdo'. Uma das Hipóteses de Solução foi analisar disciplinas relacionadas com: projeto, representação gráfica digital e gerenciamento de projeto/construção, de dois currículos de uma universidade pública brasileira. Por meio desta análise, foi possível identificar alguns parâmetros, os quais foram sistematizados em forma de um instrumento. $\mathrm{O}$ objetivo deste estudo é descrever ao leitor a estrutura do instrumento proposto e a teoria que lhe dá sustentação. O professor da disciplina a ser analisada fornece algumas informações sobre a mesma e seleciona as habilidades individuais BIM que pretende desenvolver. Ao final, é possível saber quais conceitos e usos de BIM estão relacionados com as habilidades e quais são os recursos que possivelmente serão necessários. O instrumento também informa qual é o nível de proficiência BIM da disciplina e que tipos de especialistas BIM ela pode ajudar a formar. As recomendações da ferramenta são enviadas através de mensagem eletrônica (e-mail) para o respondente e para os autores do instrumento.
\end{abstract}

Palavras-chave: Ensino de BIM. Engenharia Civil. Arquitetura e Urbanismo. Modelagem da Informação da Construção.

\begin{abstract}
The importance of teaching BIM concepts and BIM tools to students of Architecture and Civil Engineering has led several schools to introduce this subject in their curriculum. However, as in many schools the curriculum is already overloaded and there is little 'space' for including new courses, a useful strategy is to distribute BIM content over various existing courses. The purpose of this work is to propose a tool that can help teachers in planning courses with BIM content. This instrument is the outcome of a PhD research project based on all the theoretical knowledge that has been explored. In this doctoral research the 'Problematization Methodology' (PM) was employed. This follows five stages: Observation of Reality; Definition of Key Points; Theorizing; Finding possible solutions and Application to Real Situations. In the theorizing stage, 405 documents were analyzed by means of the 'content analysis' method. Another procedure was to analyze syllabuses related to design, digital graphic representation, and project management; this involved planning two different curricula for a Brazilian university. Through this analysis it was possible to classify several parameters, which could be combined in a systematic way to form an instrument. The aim of this study is to describe the framework of the proposed instrument and its underlying theory. The teacher of the course being analyzed provides some information about the course and selects the individual BIM skills that he/she intends to develop with his/her students. As a result, it is possible to find out the concepts and BIM uses related to the skills and
\end{abstract}


to determine what resources will possibly be required. The tool also gives information about the level of BIM proficiency of the course and what BIM specialists it is able to help to prepare. The recommendations of this tool are sent by email to the respondent and to the instrument authors.

Keywords: BIM Teaching. Civil Engineering. Architecture and Urbanism. Building Information Modeling

\section{INTRODUÇÃ̃O}

Este artigo apresenta uma proposta de ferramenta para auxiliar o planejamento de disciplinas com conteúdos BIM. Este instrumento é o resultado final de uma pesquisa de doutorado sobre o ensino de BIM. Na pesquisa, foi utilizada a 'Metodologia da Problematização' (MP), que possui cinco etapas: Observação da Realidade para a definição do problema de pesquisa; definição de Pontos-Chave; Teorização; Hipóteses de Solução; e Aplicação à Realidade. Na etapa de Teorização, foi utilizado o método 'Análise de Conteúdo' para se analisar 292 documentos sobre 'Ensino de BIM' e 114 documentos sobre 'Especialistas BIM'.

A análise textual dos documentos sobre ensino de BIM revelou que 168 universidades, no mundo todo, estão experimentando alguma forma de ensino de conteúdos BIM, entre as quais, 17 são do Brasil. Barison e Santos (2013) classificaram, em uma escala progressiva, denominada Nível de Proficiência BIM' (NPBIM), as várias estratégias de ensino BIM relatadas nesses documentos. Essa escala permite identificar se uma disciplina desenvolve, no aprendiz, a capacidade para dominar certos assuntos e realizar determinadas atividades que são específicas de especialistas BIM. O estudo também investigou as responsabilidades e as funções desses especialistas (BARISON, SANTOS 2010), porém, focou as competências do Gerente BIM (BARISON; SANTOS, 2011a). Succar, Sher e Williams (2013) e o Computer Integrated Construction (2012) também definiram as competências BIM individuais e de equipes, respectivamente, porém de forma genérica.

A partir dessas evidências, na etapa de levantamento de 'Hipóteses de Solução', foram determinadas algumas alternativas para se tentar solucionar o problema de pesquisa: 'encontrar formas de preparar estudantes de Arquitetura e de Engenharia Civil para ingressar no mercado de trabalho com conhecimentos e habilidades em BIM'. Na etapa de 'Aplicação à Realidade', foram escolhidas as soluções possíveis de serem encaminhadas para que a realidade possa ser transformada em algum grau.

Uma das várias soluções propostas pelo estudo, possível de ser implementada, envolveu a análise dos currículos de dois cursos de uma universidade pública do Estado de São Paulo: Arquitetura e Urbanismo e Engenharia Civil. O objetivo foi identificar disciplinas que possuem potencial para ensinar BIM. Entretanto, para realizar a análise das ementas desses dois currículos, foi necessário utilizar alguns parâmetros, os quais foram sistematizados em forma de uma ferramenta computacional.

Portanto, o objetivo deste trabalho é apresentar esta ferramenta que pode ser utilizada não apenas pelos professores desses dois cursos, como também por professores de outras universidades. Vale ressaltar que uma das limitações da ferramenta é a plataforma escolhida para sua implementação piloto, que não ofereceu alguns recursos necessários, e que poderão ser aprimorados em implementação posterior. 


\section{A IMPLEMENTAÇÃO DA FERRAMENTA}

\subsection{Justificativa}

Devido à importância de se ensinar conceitos e ferramentas BIM para estudantes de Arquitetura e Urbanismo e de Engenharia Civil, várias escolas têm procurado introduzir este assunto em seus currículos. No entanto, como o currículo já está sobrecarregado e há pouco espaço para novas disciplinas, uma estratégia útil é distribuir conteúdos BIM em diversas disciplinas do curso.

Com o objetivo de transformar a realidade em algum grau, esta pesquisa investigou dois currículos de uma universidade pública do Estado de São Paulo. A investigação envolveu a análise textual de ementas de disciplinas, assim como as respostas a um questionário que foi aplicado a professores das disciplinas selecionadas. Como resultado, foi possível identificar alguns parâmetros, os quais foram, posteriormente, sistematizados em forma de um instrumento.

\subsection{Interface}

A ferramenta proposta é um questionário on-line que fornece recomendações de acordo com as respostas fornecidas pelo usuário.

Para implementar esse questionário, foi utilizado o Google Forms. O professor fornece informações sobre a disciplina a ser analisada e seleciona as habilidades BIM que pretende desenvolver. Ao final, é possível saber: quais conceitos e usos de BIM estão relacionados com as habilidades BIM selecionadas, qual é o NPBIM recomendado para a disciplina e que tipo de especialista BIM a disciplina ajudaria a formar. $\mathrm{O}$ instrumento envia duas mensagens eletrônicas com recomendações: uma para o respondente e outra para os autores.

Inicialmente, o respondente fornece as seguintes informações: nome da universidade, nome do curso e nome da disciplina. Em seguida, o respondente seleciona os conhecimentos prévios dos alunos que cursarão a disciplina (Quadro 1). A partir desta seleção, a ferramenta informa qual é o NPBIM recomendado para a disciplina e solicita que o usuário selecione as habilidades que ele pretende desenvolver.

\section{Quadro 1 - Conhecimentos prévios dos alunos}

\begin{tabular}{ll}
\hline Bloco & Conhecimentos prévios \\
\hline 1 & Não possuem experiência em ferramentas BIM \\
& Não conhecem conceitos BIM \\
& Não possuem conhecimentos na área de AEC \\
\hline 2 & Têm experiência em uma ferramenta BIM de sua área \\
& Conhecem conceitos de BIM \\
& Conhecem conceitos básicos de modelagem de dados \\
& Sabem gerar documentações, quantidades e especificações a partir do modelo BIM \\
& Conhecem fundamentos de projeto \\
& Sabem comunicar informações de projetos de arquitetura/engenharia \\
\hline 3 & Têm experiência na utilização das principais ferramentas BIM \\
& Conhecem técnicas avançadas de modelagem 3D \\
& Conhecem padrões BIM e fluxo de trabalho BIM \\
& Sabem utilizar um modelo BIM em processos 4D e 5D \\
& Têm experiência profissional em AEC \\
& Conhecem linguagem de programação \\
& Conhecem materiais e métodos de construção
\end{tabular}




\begin{tabular}{ll}
\hline & Conhecem tecnologia de construção \\
Conhecem sistemas do edifício
\end{tabular}

No total, existem no instrumento 235 habilidades BIM individuais distribuídas em 8 grupos e 51 subgrupos. Ao usuário são apresentadas somente as habilidades referentes ao NPBIM menor ou igual ao NPBIM recomendado para a disciplina (Tabela 1).

\section{Tabela1 - Quantidade de Habilidades BIM de cada NPBIM}

\begin{tabular}{lll}
\hline NPBIM & Quantidade de Habilidades $(\mathbf{N}=235)$ & Habilidades visíveis \\
\hline Introdutório & 9 & 9 \\
Intermediário & 46 & $46+9=55$ \\
Avançado & 28 & $46+9+28=83$ \\
Profissional & 152 & $46+9+28+152=235$ \\
Total & 235 & \\
\hline
\end{tabular}

A identificação das habilidades (Quadro 2) baseou-se em Succar, Sher e Williams (2013), no Computer Integrated Construction (2011) e em Barison e Santos (2011a).

Quadro 2 - Agrupamentos de habilidades BIM

\begin{tabular}{llll}
\hline $\mathbf{N}$ & Grupo (N=8) & N & Subgrupo (N=51) \\
\hline 1 & Técnicas & 1 & Habilidades técnicas Básicas \\
& 2 & Gerenciamento de documentos \\
& 3 & Específicas com equipamentos \\
& 4 & Gerenciamento do Modelo \\
& 5 & Modelagem e desenhos \\
\hline 2 & Gerenciais & 6 & Detalhamento e documentação \\
& 7 & Gerenciamento geral \\
& 8 & Gerenciamento estratégico \\
& 9 & Gerenciamento organizacional \\
& 10 & Gerenciamento do cliente \\
& 11 & Parcerias e alianças \\
\hline 3 & Administrativas & 12 & Liderança \\
& 13 & Finanças, custos e orçamentos \\
& 14 & Administração geral \\
& 15 & Procedimentos e políticas do escritório \\
& 16 & Recursos humanos e recrutamento \\
& 17 & Marketing \\
& 18 & Licitações e aquisições \\
& 19 & Observância e administração de contratos \\
& 20 & Gerenciamento de risco \\
& 21 & Controle de qualidade \\
\hline 4 & Funcionais & 22 & Colaboração \\
& 23 & Habilidades funcionais básicas \\
& 24 & Gerenciamento do fluxo de trabalho e equipe \\
& 25 & Gerenciamento do empreendimento \\
\hline 5 & Operacionais & 26 & Planejamento e programação \\
& 27 & Quantificação e estimativa \\
& 28 & Uso geral do modelo \\
& &
\end{tabular}




\begin{tabular}{llll} 
& 29 & Análises e Simulaçães \\
& 30 & Seleção e Especificação \\
31 & Verificação e Monitoramento \\
32 & Operação e Controle \\
& 33 & Ligação e extensão \\
& 34 & Concepção e conceitualização \\
\hline 6 & De implantação & 35 & Desenvolvimento de componente \\
& 36 & Guias e Manuais \\
& 37 & Fundamentos de implementação \\
& 38 & Padronização e templates \\
& 39 & Testagem do processo e sistema \\
& 40 & Treinamento técnico \\
& 41 & Gerenciamento da biblioteca \\
\hline 7 & De suporte & 42 & Software e projeto de web \\
& 43 & Dados, arquivos e gerenciamento da rede \\
& 44 & Suporte TI em geral \\
& 45 & Resolução de problemas com software \\
& 46 & Facilitação de mudanças \\
& 47 & Habilidades de avaliação e pesquisa \\
& 48 & Planejamento e desenvolvimento de estratégia \\
& 49 & Ensino e treinamento \\
& 50 & Desenvolvimento e pesquisa geral \\
& 51 & Engenharia do conhecimento \\
\hline
\end{tabular}

Fonte: Succar, Sher e Williams (2012); Barison e Santos (2011a) e Computer Integrated Construction (2011)

Depois que o usuário remete sua resposta, a ferramenta processa as informações nela contidas e faz algumas recomendações úteis. Por exemplo, de acordo com as habilidades selecionadas, a ferramenta determina um grupo de conceitos (Quadro 3) e um grupo de usos de BIM (Quadro 4) relacionados a eles. Além disso, a ferramenta indica o nível cognitivo de cada habilidade, dos conceitos e usos, conforme o NPBIM recomendado.

\section{Quadro 3 - Conceitos relacionados ao BIM e níveis cognitivos recomendados}

\begin{tabular}{|c|c|c|c|c|c|}
\hline $\mathbf{N}$ & Conceitos relacionados ao BIM & $\begin{array}{l}1^{\circ} \text { e } 2^{\circ} \text { anos } \\
\text { Introdutório }\end{array}$ & $\begin{array}{l}3^{\circ} \text { e } 4^{0} \text { anos } \\
\text { Intermediário }\end{array}$ & $\begin{array}{l}5^{\circ} \text { ano } \\
\text { Avanç. }\end{array}$ & $\begin{array}{l}\text { Profis. } \\
\text { Profiss. }\end{array}$ \\
\hline 1 & Modelagem paramétrica & Aplicar & Aplicar & Avaliar & Criar \\
\hline 2 & $\begin{array}{l}\text { Propriedade intelectual do modelo e } \\
\text { componentes }\end{array}$ & Entender & Entender & Aplicar & Analisar \\
\hline 3 & Programação Orientada a Objetos & Entender & Aplicar & Analisar & Avaliar \\
\hline 4 & $\begin{array}{l}\text { Modelagem Generativa e Geometria } \\
\text { Complexa }\end{array}$ & Entender & Aplicar & Avaliar & Criar \\
\hline 5 & Integridade e Consistência de dados & Entender & Entender & Aplicar & Criar \\
\hline 6 & $\begin{array}{l}\text { Restrições e regras que definem } \\
\text { comportamentos de objetos }\end{array}$ & Entender & Aplicar & Avaliar & Criar \\
\hline 7 & Interoperabilidade & Entender & Entender & Aplicar & Criar \\
\hline 8 & Padronização BIM & Entender & Entender & Aplicar & Analisar \\
\hline 9 & $\begin{array}{l}\text { Novas formas de contratos (inclui } \\
\text { IPD) }\end{array}$ & Entender & Aplicar & Analisar & Avaliar \\
\hline 10 & Fluxo de trabalho BIM & Entender & Aplicar & Analisar & Avaliar \\
\hline 11 & $\begin{array}{l}\text { Nível de detalhe e nível de } \\
\text { desenvolvimento do modelo }\end{array}$ & Não se aplica & Aplicar & Analisar & Avaliar \\
\hline 12 & Segurança de dados & Não se aplica & Entender & Aplicar & Criar \\
\hline
\end{tabular}


13 Aspectos legais e contratuais da implementação BIM

14 Repositório de informações e base central de dados

15 Ferramentas de comunicação e mídia

16 Compartilhamento de informações

17 Seleção e avaliação de ferramentas, processos e/ou tecnologias BIM

18 Mudanças em processos e procedimentos de gestão

19 Vantagens de BIM para projeto e construção e riscos associados

20 Mudança de papéis

21 Obstáculos na implementação BIM

22 Nível de maturidade e nível de capacidade BIM de organizações

23 Colaboração

24 Gerenciamento do fluxo de informações

25 Procedimentos de contratação/gestão

\begin{tabular}{|c|c|c|c|}
\hline Não se aplica & Entender & Aplicar & Analisar \\
\hline Não se aplica & Entender & Analisar & Criar \\
\hline Aplicar & Aplicar & Criar & Avaliar \\
\hline Aplicar & Aplicar & Aplicar & Avaliar \\
\hline Não se aplica & Aplicar & Criar & Avaliar \\
\hline Não se aplica & Aplicar & Aplicar & Criar \\
\hline Não se aplica & Aplicar & Analisar & Criar \\
\hline Não se aplica & Entender & Aplicar & Analisar \\
\hline Não se aplica & Entender & Aplicar & Analisar \\
\hline Não se aplica & Entender & Aplicar & Analisar \\
\hline Não se aplica & Entender & Aplicar & Analisar \\
\hline Não se aplica & Entender & Aplicar & Analisar \\
\hline Não se aplica & Aplicar & Analisar & Avaliar \\
\hline
\end{tabular}

Obs. Níveis em negrito foram adaptados da pesquisa Sacks e Pikas (2013).

A ferramenta também gera um gráfico que ajuda o usuário a visualizar quantas das habilidades de cada especialista BIM e respectivos tipos de profissionais são desenvolvidas na disciplina, conforme pode ser observado no exemplo da Figura 1.

\section{Figura 1 - Gráfico de habilidades dos especialistas e profissionais}

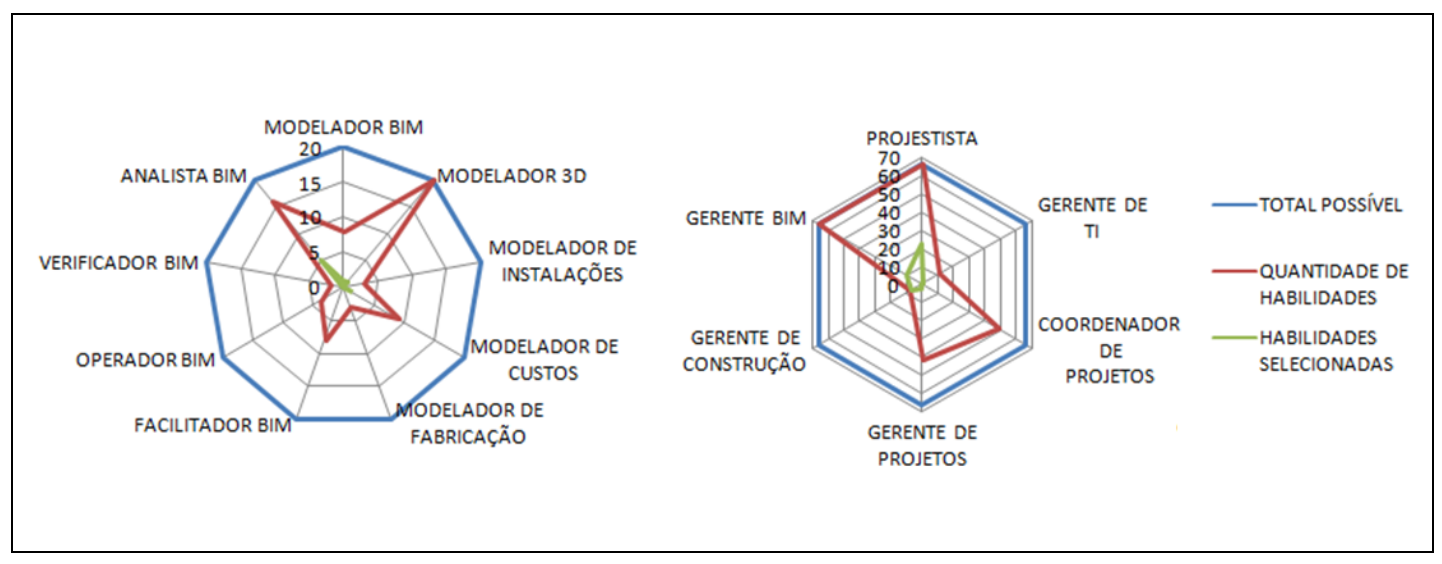

Por fim, a ferramenta informa as características do NPBIM recomendado para a disciplina, em termos de: tipos de projetos que podem ser conduzidos e tipos de colaboração que podem ser praticados (BARISON; SANTOS 2011b). Além disso, sugere recursos de acordo com os usos de BIM relacionados.

\section{Quadro 3 - Usos de BIM}

\begin{tabular}{llcccc}
\hline $\mathbf{N}$ & Usos de BIM & $\begin{array}{c}\mathbf{1}^{\mathbf{0}} \mathbf{e} \mathbf{2}^{\mathbf{0}} \\
\text { Introdutório }\end{array}$ & $\begin{array}{c}\mathbf{3}^{\mathbf{o}} \mathbf{e} \mathbf{4}^{\mathbf{0}} \\
\text { Intermed. }\end{array}$ & $\begin{array}{c}\mathbf{5}^{\mathbf{0}} \text { ano } \\
\text { Avanç. }\end{array}$ & $\begin{array}{c}\text { Profiss. } \\
\text { Profiss. }\end{array}$ \\
\hline 1 & Modelagem de condições existentes & Aplicar & Aplicar & Analisar & Avaliar \\
2 & Estimativa de custos automatizada & Aplicar & Aplicar & Analisar & Avaliar \\
3 & Integração de BIM com ERP & Não se aplica & Entender & Entender & Aplicar
\end{tabular}



customizados

11 Modelagem de sólido/volume

12 Geração de ambientes imersivos

13 Criação de renderizações e apresentações

14 Produção de desenhos - detalhes em 3D

15 Geração automatizada - documentação

16 Verificação do código de obras

17 Verificação da conformidade com os requisitos do programa/cliente

18 Análise energética

19 Análise estrutural

20 Análise de iluminação

21 Análise de ar-condicionado

22 Análises e revisão de construtibilidade

23 Simulação de evento discreto

24 Avaliação de sustentabilidade (LEED, etc.)

25 Outras análises de engenharia

26 Detecção de interferências

27 Coordenação 3D

28 Construção virtual

29 Fabricação/instalação controlada por computador

30 Planejamento da utilização do canteiro

31 BIM na obra

32 Planejamento e controle 3D

33 Modelo de registro BIM

34 Gerenciamento/manutenção da facilidade

35 Rastreamento/gerenciamento de espaços

36 Gerenciamento do patrimônio

37 Análises de sistemas do edifício

38 Planejamento de desastres

\begin{tabular}{|c|c|c|c|}
\hline Aplicar & Aplicar & Analisar & Avaliar \\
\hline Aplicar & Aplicar & Analisar & Criar \\
\hline Aplicar & Aplicar & Analisar & Avaliar \\
\hline Aplicar & Aplicar & Analisar & Avaliar \\
\hline Não se aplica & Entender & Aplicar & Analisar \\
\hline Aplicar & Aplicar & Aplicar & Analisar \\
\hline Aplicar & Aplicar & Analisar & Criar \\
\hline Aplicar & Aplicar & Analisar & Avaliar \\
\hline Não se aplica & Entender & Aplicar & Analisar \\
\hline Entender & Entender & Entender & Analisar \\
\hline Não se aplica & Aplicar & Analisar & Avaliar \\
\hline Entender & Entender & Aplicar & Analisar \\
\hline Entender & Entender & $\mathbf{X}$ & Analisar \\
\hline Entender & Entender & $\mathbf{x}$ & Analisar \\
\hline Entender & Entender & Entender & Analisar \\
\hline Entender & Entender & Entender & $\mathbf{x}$ \\
\hline Não se aplica & Aplicar & Analisar & Avaliar \\
\hline Não se aplica & Aplicar & Analisar & Avaliar \\
\hline Aplicar & Aplicar & Analisar & Avaliar \\
\hline Não se aplica & Não se aplica & Entender & $\mathbf{X}$ \\
\hline Não se aplica & Não se aplica & Aplicar & Avaliar \\
\hline Não se aplica & Entender & Entender & $\mathrm{X}$ \\
\hline Não se aplica & Aplicar & Analisar & Avaliar \\
\hline Entender & Entender & Aplicar & Avaliar \\
\hline Não se aplica & Entender & Entender & Aplicar \\
\hline Entender & Entender & Aplicar & Analisar \\
\hline Aplicar & Aplicar & Analisar & Avaliar \\
\hline Entender & Aplicar & Analisar & Criar \\
\hline Entender & Entender & Aplicar & Analisar \\
\hline Entender & Entender & Aplicar & Analisar \\
\hline Entender & Entender & Entender & Aplicar \\
\hline Entender & Entender & Entender & Aplicar \\
\hline Entender & Entender & Entender & Aplicar \\
\hline Não se aplica & Entender & Entender & Aplicar \\
\hline Não se aplica & Entender & Entender & Aplicar \\
\hline
\end{tabular}

Obs. Níveis em negrito foram adaptados da pesquisa Sacks e Pikas (2013) para a Taxonomia de Bloom revisada.

Fonte: Adaptado de Sacks e Pikas (2013) e Computer IntegratedConstruction (2011)

\section{A FERRAMENTA}

\subsection{Fluxograma}

A Figura 2 apresenta um fluxograma da ferramenta. Nele, é possível observar a entrada de informações pelo usuário e o processamento das recomendações.

Inicialmente, o usuário seleciona os conhecimentos prévios dos alunos, os quais determinarão o NPBIM recomendado. Este, por sua vez, determinará quais habilidades BIM estarão visíveis e são passíveis de ser selecionadas pelo usuário. A desativação de habilidades não recomendadas, tanto facilita a seleção pelo respondente pela redução da quantidade de opções, quanto o orienta ao não permitir escolha de habilidades que dependem de pré-requisitos que os alunos ainda não têm. Após submeter suas escolhas, o usuário recebe um e-mail com as recomendações. 
As recomendações descrevem as características do NPBIM sugerido, em termos de: tipo de colaboração e projeto a ser desenvolvido pelos alunos. Em seguida, são apresentadas três tabelas: lista das habilidades BIM selecionadas pelo usuário, lista de conceitos BIM ligados às habilidades e lista de usos de BIM relacionados a elas. Além disso, para cada habilidade, conceito e uso há uma recomendação sobre o nível cognitivo a ser alcançado na disciplina (Quadro 4). Com estas informações, o professor pode escolher quais usos de BIM abordar na disciplina e, quais atividades educacionais planejar, em função do nível cognitivo. Os gráficos de especialistas BIM e respectivos profissionais são gerados de acordo com o especialista BIM indicado para cada habilidade.

Figura 2 - Fluxograma da ferramenta

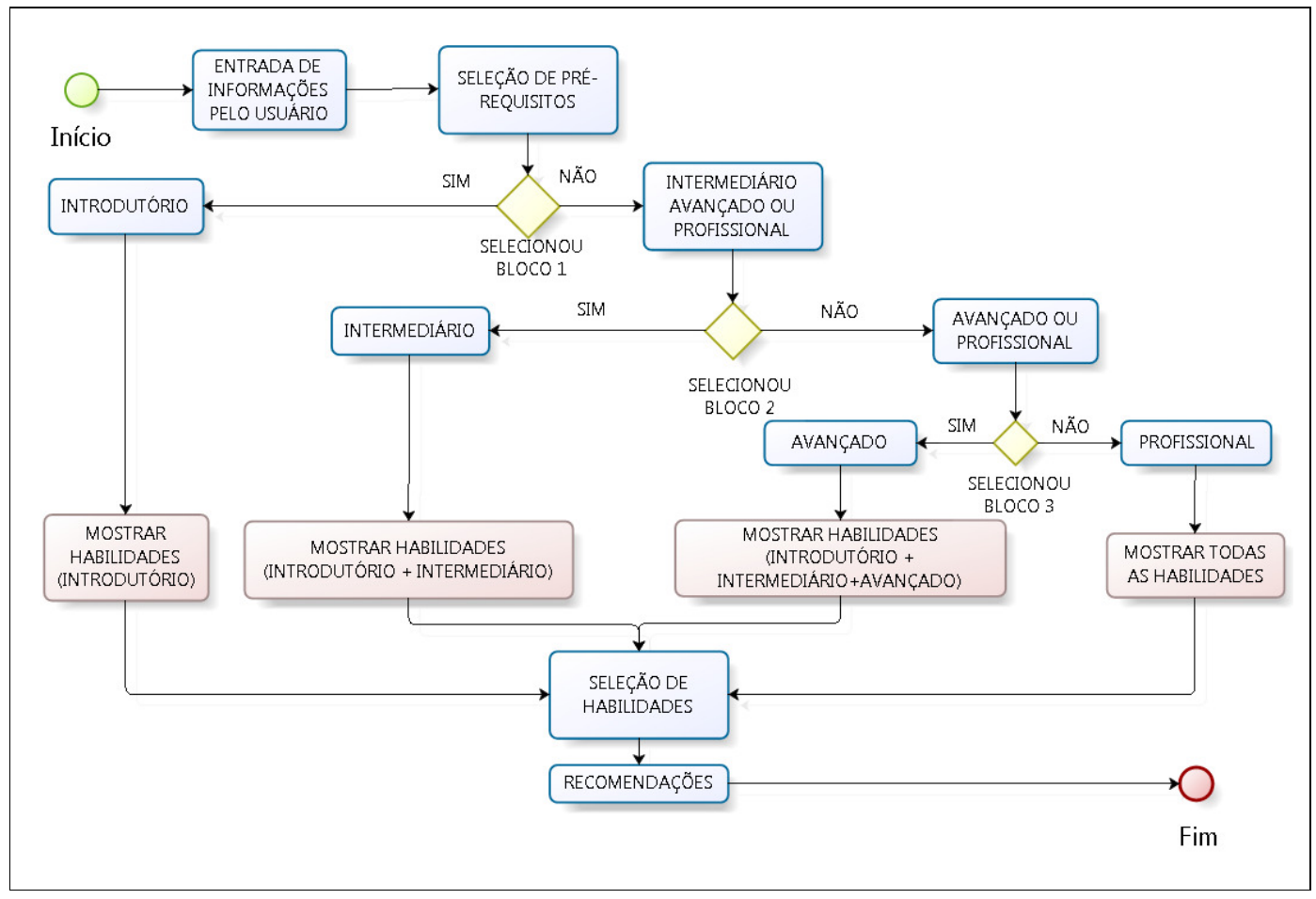

Fonte: Os autores, 2014

\section{Quadro 4 - Taxonomia de Bloom revisada}

\begin{tabular}{ll}
\hline Nível cognitivo & Descrição \\
\hline Lembrar & $\begin{array}{l}\text { Consegue reconhecer e recordar informações importantes usando a } \\
\text { memória de longa duração. }\end{array}$ \\
Entender & $\begin{array}{l}\text { É capaz de fazer sua própria interpretação do material. } \\
\text { Aplicar }\end{array}$ \\
$\begin{array}{l}\text { É capaz de usar o conhecimento aprendido em uma situação nova ou } \\
\text { familiar. }\end{array}$ \\
Enalisar & $\begin{array}{l}\text { É capaz de dividir o todo em partes e descobrir como essas partes se } \\
\text { relacionam com o todo. }\end{array}$ \\
Avaliar & É capaz de verificar e criticar. \\
Criar & É capaz de reunir elementos para dar origem a algo novo. \\
\hline
\end{tabular}




\subsection{Princípios das recomendações}

A análise textual dos 114 documentos sobre 'Especialistas BIM' mostrou que há uma ampla variedade de funções que requer um conjunto de habilidades. Na prática, um profissional pode conduzir as tarefas de um ou mais especialistas, dependendo do grau de complexidade do projeto e do tamanho da empresa em que ele/ela trabalha.

Cada competência BIM individual (Quadro 1) é relacionada com seu respectivo especialista. O objetivo é informar ao usuário qual especialista BIM a disciplina irá abordar.

A matriz NPBIM consiste de três níveis: introdutório, intermediário e avançado. Em cada nível existe um grupo de características que mostra a natureza do projeto BIM a ser conduzido pelos estudantes. Um quarto nível foi adicionado à ferramenta: profissional.

Sacks e Pikas (2013) também conceberam uma metodologia para que escolas de AEC pudessem definir o tipo de conteúdo BIM a ser ensinado. Esta metodologia envolveu uma pesquisa com profissionais e resultou em uma lista de 39 competências BIM individuais, relacionadas à Taxonomia de Bloom. O presente estudo utilizou os resultados da pesquisa de Sacks e Pikas (2013) para determinar o relacionamento entre níveis cognitivos e NPBIM. Contudo, no instrumento aqui apresentado, foi utilizada a Taxonomia de Bloom revisada, cujo nível mais alto é "criar" ao invés de "avaliar".

Um estudo do Computer Integrated Construction (2011) determinou os recursos requeridos para se trabalhar com cada uso de BIM. O presente estudo baseou-se nas recomendações do Computer Integrated Construction (2011) e na matriz de ferramentas BIM publicada pelo BIM Fórum (2011). Portanto, o instrumento aqui apresentado agregou esses conhecimentos em uma interface fácil de usar, acessível ao professor não especialista em ensino de BIM.

\subsection{Validação do instrumento}

Todo o conteúdo relacionado a habilidades, conceitos, usos e recursos foi revisado por três profissionais que estão praticando BIM e que possuem respeitabilidade, experiência de trabalho e liderança. A abordagem foi qualitativa porque, no Brasil, existem ainda poucos especialistas nesta área. Os profissionais corrigiram o conteúdo dos quadros e deram sugestões.

\section{CONCLUSÕES}

O instrumento foi implementado ${ }^{1}$ como um aplicativo da Web para uso on-line (GoogleForms). A recomendação fornecida pelo instrumento fundamenta-se nas conclusões da pesquisa de doutorado, desenvolvida pelos autores, sobre o planejamento de uma disciplina BIM e/ou currículo. Acredita-se que, com base nesse conhecimento, o usuário poderá avaliar, com maior facilidade, a adequação dessa proposta para o caso particular de sua disciplina.

Esta pesquisa de doutorado procura sintetizar conhecimentos advindos de vários importantes autores, em sua maioria internacional, nas áreas: ensino de BIM e especialistas BIM. Com esse trabalho, parte desse conhecimento é disponibilizada em um formato possível de ser usado pelos interessados primários (professores

\footnotetext{
${ }^{1}$ A implementação da ferramenta foi realizada por um aluno do $3^{\circ}$ ano do curso de Ciências da Computação - UEL (Felipe Kengi Nakano), sob a orientação do Prof. Dr. Fabio Sakuray.
} 
universitários dos cursos de Arquitetura e Engenharia Civil, de forma simples e semiautomatizada.

Ao se levar em consideração o longo tempo de existência da ampla maioria dos cursos superiores nas áreas de AEC, pode-se concluir que cursos focados em BIM ou que abordam esse tema ainda são extremamente recentes nas Universidades. No entanto, a demanda do mercado por profissionais qualificados por universidades já é bastante significativa. Mais que isso, o próprio sucesso da implantação mais ampla de BIM em um país dependerá da resposta de suas universidades em relação a essa formação, pois profissionais qualificados atuam como agentes de transformação da cultura e da tecnologia empregadas na Construção.

\section{AGRADECIMENTOS}

Os autores agradecem à CAPES e ao CNPq pelo apoio recebido nesta pesquisa, e à Fundação de Amparo à Pesquisa do Estado de São Paulo (FAPESP), processo $n^{\circ}$ 2014/16362-1, pelo apoio em sua divulgação.

\section{REFERÊNCIAS}

ANDERSON, L.W. et al.ATaxonomy for learning, teaching, and assessing: A revision of Bloom's Taxonomy of Educational Objectives.New York: Longman, 2001 .

BARISON, M. B., SANTOS, E. T. An overview of BIM specialists. In: INTERNATIONAL CONFERENCEON COMPUTINGIN CIVILAND BUILDING ENGINEERING, Proceedings...W. Tizani, ed., Nottingham University Press, Nottingham, UK, 2011 p.141.

The competencies of BIM specialists: A comparative analysis of the literature review and job ad descriptions. Proceedings...IN: INTERNATIONAL WORKSHOPON COMPUTINGIN CIVIL ENGINEERING, ASCE, Reston, VA, 2011a.

BIM Teaching: Current International Trends.Gestão e Tecnologia de Projetos, v. 6, n. 2, p. 67-80. 2011.

BIM FÓRUM (2011). BIM Matrix Tools. Disponível em: <http://bimforum.org/wpcontent/uploads/2011/02/BIM_Tools_Matrix.pdf> Acesso em: 20 dez. 2013.

COMPUTER INTEGRATED CONSTRUCTION. BIM Project Execution Planning Guide.Disponível em: <http://bim.psu.edu/Uses/> Acesso em: 11 dez. 2013.

SACKS, R., PIKAS, E. Building Information Modeling Education for Construction Engineering and Management: Industry Requirements, State of the Art, and Gap Analysis. J. Constr. Eng. Manage, v.139, n. 11, 2013.

SUCCAR, B., SHER, W., WILLIAMS, A. An integrated approach to BIM competency assessment, acquisition and application. Automation in Construction,v.35, p. 174-189, 2013. 